\title{
Vaccines in Non-Small Cell Lung Cancer
}

\author{
Luis Corrales-Rodriguez ${ }^{1}$, Normand Blais ${ }^{2}$, Denis Soulières ${ }^{2}$ \\ ${ }^{1}$ Hospital San Juan de Dios, San José, Costa Rica; ${ }^{2}$ Centre Hospitalier de l’Université de Montréal, Hopital Notre-Dame, Montréal, \\ Canada. \\ Email: lcorralesr@yahoo.com
}

Received October $18^{\text {th }}, 2012$; revised November $18^{\text {th }}, 2012$; accepted November $28^{\text {th }}, 2012$

\begin{abstract}
Immunotherapy as an option of treatment in cancer has experienced an important development with the inclusion of vaccines. In lung cancer this type of treatment has emerged and vaccines can be classified in three groups: antigen-specific vaccines, tumor cell vaccines, and dendritic cell vaccines. Emepepimut (L-BLP25) and MAGE-A3 have been the vaccines most widely studied. Their promising results with benefit in survival and limited toxicity in preclinical and clinical trials have led to phase III trials with results eagerly awaited. Other vaccines have been investigated, but results were not favorable or are still pending. Hopefully, vaccines could be an additional instrument for the treatment of lung cancer in the adjuvant or metastatic setting as time will unveil the results of current and future trials.
\end{abstract}

Keywords: Emepepimut-S; Immunotherapy; Lung Cancer; MAGE-A3; NSCLC; Review; Vaccine

\section{Introduction}

The human immune system comprehends a complex interrelation of pathways that provide different responses. Inflammation is one of these important responses the human body originates by trying to remove any deleterious stimuli and initiating the corresponding healing process [1].

In 1863, Virchow hypothesized that cancer was preceded by chronic inflammation due to an enhanced cell proliferation [2]. Today, the relationship between inflammation, innate immunity and cancer is widely accepted, but understanding the role cellular and molecular mechanisms play in this relationship is complex and not clearly understood [3].

The mechanisms of cellular immunity are started as antigen-presenting cells (APC), which include macrophages and dendritic cells, internalize circulating antigens. These cells create short peptides that are exposed at their surface in association with the major histocompatibility complex (MHC) class II molecule. As APC circulate, they come in contact with the appropriate CD4+ Thelper lymphocyte which then activates specific effector CD8+ cytotoxic T lymphocytes. These cytotoxic lymphocytes will circulate and recognize altered cells displaying the complementary MHC class I molecule on the surface, leading to granule exocytosis or expression of FAS ligand which will activate apoptosis [4,5].

Unfortunately, tumors may "evade" the normal human immune response, leading to development and proliferation of the disease. Given that tumors originate from the same organism from which the immune response will develop, tumors might be poorly immunogenic. Also, tumors might not activate apoptosis, down-regulate antigen expression, and secrete peptides that might lead to [6] tolerance or inhibition of the immune response [4,7].

Lung cancer has the highest cancer related mortality in the world with a 5 years overall survival of 15\% [8,9]. Non-small cell lung cancer subtype accounts for the majority of the lung cancers, accounting $80 \%$ of cases [10]. Given the increasing frequency and the high mortality, new chemotherapeutic regimens and specific target compounds have been approved to treat this disease, with important advances in response and survival [11-14]. Still, lung cancer incidence and mortality are very high, though new and innovative treatment approaches are necessary.

Vaccines for cancer have been under development during the past years. Sipuleucel-T (Provenge ${ }^{\circledR}$; Dendreon) was the first therapeutic vaccine approved by the FDA in April 2010, targeting prostate cancer $[15,16]$. Sipuleucel-T induces an immune response targeted against the prostatic acid phosphatase, an antigen commonly expressed in prostate cancer. This type of immune response promotes an additional and targeted therapeutic option in prostate cancer, but the mechanisms by which Sipuleucel-T acts are somewhat similar to those that can be developed to treat other cancers.

Vaccines in cancer can be subdivided in antigen-specific, tumor cell, and dendritic cell vaccines. Of these, dendritic cell vaccines have not been widely developed 
in NSCLC $[4,17]$.

NSCLC has benefited from the development of immunotherapy, and various molecules are currently under investigation. Emepepimut-S (Stimuvax ${ }^{\circledR}$; Merck Serono) and MAGE-A3 (GlaxoSmithKline) are the vaccines that have shown the most promising results, but others are still under study.

\section{Emepepimut-S}

Emepepimut-S (Stimuvax ${ }^{\circledR}$; Merck Serono) is an antigen-specific liposomal vaccine that targets the exposed core peptide of MUC1 tumor associated antigen, inducing a cellular immune response. This response leads to rejection of cells that express MUC1 [18]. Also called BLP25 (Biomira Liposomal Peptide 25), the vaccine consists of a 25-amino acid sequence that contains a palmitoyl lysine residue at the carboxyl terminal to help incorporate the lipopeptide into the liposome particle, monophosphoryl lipid A, and three lipids (cholesterol, dimyristoyl phosphatidylglycerol, and dipalmitoyl phosphatidylcholine) [19]. Studies have shown there is a strong antigen-specific $\mathrm{T}$ lymphocyte proliferation with a rapid response of cytotoxic $\mathrm{T}$ lymphocytes and helper $\mathrm{T}$ lymphocytes, generating an anti-MUC1 cell response towards cells expressing this protein [20].

Two phase 1 studies have been published using emepepimut-S in NSCLC patients with stage IIIB and IV. Doses administered varied between them, one including doses of $20 \mu \mathrm{g}$ or $200 \mu \mathrm{g}$ for a total of 4 treatments at weeks $0,2,5$, and 9 , and the other using a dose of 1000 $\mu \mathrm{g}$ every week for 8 weeks followed by maintenance doses every 6 weeks, with a single cyclophosphamide dose 3 days before the L-BLP25 administration. Results revealed stabilization of the disease, adequate tolerance, and a cellular immune response, giving rise to phase II studies [21]. Due to the interesting results of the previous studies, a phase IIB open-label study was designed to detect a difference in survival of 5 months with a power of $80 \%$. Non-small cell lung cancer stage IIIB and IV patients were enrolled between 2000 and 2002 by centers of Canada and the United Kingdom, including a total of 171 patients. 88 patients were admitted in the study arm and the rest were part of the BSC arm. BLP-25 liposome vaccine (L-BLP25) was administered weekly at a dose of $1000 \mu \mathrm{g}$ (total dose was administered subcutaneously in 4 divided doses) during eight weeks and patients could be placed in maintenance therapy which consisted of vaccinations every six weeks starting on week 13 [22]. Patients in the immunotherapy arm were administered a single intravenous dose of cyclophosphamide 3 days before the vaccine since previous studies showed an increased immunotherapeutic effect through various mechanisms $[23,24]$. Results from this phase II study showed a non-significant prolongation of the me- dian survival time of 4.4 months (HR $=0.739$; 95\% CI, 0.509 - 1.073) and the greatest benefit was seen in patients with stage IIIB locoregional disease.

There is another phase II study which included 22 patients with unresectable stage IIIA-IIIB NSCLC. These patients were treated with a different formulation of L-BLP25, where changes to the lipid A acyl chain composition were made. The drug was given at a dose of $1000 \mu$ g every week for a total of 8 weeks followed by a maintenance dose of $1000 \mu \mathrm{g}$ every 6 weeks starting at week 13 until there was evidence of disease progression. After a follow-up of 26.7 months, 1-year survival rate was $82 \%$ (95\% CI; $66 \%$ - 98\%) and the 2-year survival rate was $64 \%$ (95\% CI; 44\% - 84\%) [25].

There are no phase III studies published with L-BLP25. Phase III studies have been opened, analyzing the role of in lung cancer. A placebo-controlled randomized Asian study (INSPIRE: Stimuvax Trial in Asian NSCLC Patients: Stimulating Immune Response) is currently recruiting stage III unresectable NSCLC patients with stable disease or who responded to primary chemoradiotherapy [26]. Also, the Stimulating Targeted Antigenic Responses to NSCLC (START) multinational trial has been started. It will evaluate L-BLP-25 as maintenance therapy in patients diagnosed with unresectable stage III NSCLC, aiming to enroll more than 1300 patients. Overall survival and safety will be assessed [27].

Safety of emepepimut-S has been confirmed in the various trials using the vaccine. In the phase II study by Butts et al., no significant toxicity was observed and quality of life was maintained for a longer period of time in those patients who were treated with L-BLP25. 98.9\% of the L-BLP25 arm and $95.2 \%$ of the control arm reported adverse events, mostly disease related, and $28.4 \%$ of the adverse events in the exploratory arm were considered to be due to the cyclophosphamide administered. Grade 1 flu-like syndrome and injection site reactions were the most common events in the exploratory arm, and serious adverse events were described in $26.1 \%$ of patients in the study arm (36.1\% in the BSC arm), but only an episode of pneumonia was linked to the study drug [25]. In a long term safety analysis, 16 patients received the drug for a period of 2.0 to 7.7 years with a $96 \%$ compliance rate during the maintenance treatment given every 6 weeks. The most common treatment associated adverse events reported by the authors were cough ( $n=12)$, fatigue $(n=12)$, and dyspnea $(n=11)$, but there was no increase in the frequency of adverse events due to the time of exposure of the treatment. No long-term renal or liver toxicity was observed, and no adverse event related to autoimmunity was encountered [28].

The analysis of adverse events from the phase II study using a monophosphoryl lipid $\mathrm{A}$ in the preparation of L-BLP25 reported that the grade $1 / 2$ adverse events were 
similar to those reported in the previous studies. These adverse events included fatigue, dyspnea, insomnia, anorexia, headache, diarrhea, paresthesia, abdominal pain, influenza-like illness, urinary tract infection and peripheral neuropathy. No discontinuation of treatment was observed [25,29]. In a multiple myeloma exploratory phase II study using L-BLP25, a patient developed an episode of encephalitis. This patient was exposed to an intensified low-dose cyclophosphamide, not regularly used in other studies, which led to a temporary suspension of clinical trials that included L-BLP25. This suspension was later revoked and trials, such as INSPIRE and START, were restarted [30].

In general, the safety of the vaccine has been demonstrated in various studies, and in general, the quality of life of patients receiving the vaccine has not been affected.

\section{MAGE-A3}

The Melanoma Associated Antigen (MAGE)-A gene family is expressed in cancer cells but not in normal tissue, therefore providing a therapeutic opportunity. MAGE-A3 produces an antigenic nonapeptide recognizable by $\mathrm{T}$ cells on the HLA-A1 molecule [31]. MAGE-A3 is expressed in around 35\% of NSCLC patients and probably is related to a poor prognosis [32]. Initially developed against melanoma, the vaccine proved to have some response in these patients with a CD4 T-lymphocyte response directed to MAGE-A3 antigen in one patient [33].

MAGE-A3 vaccine is an antigen-specific vaccine evaluated in a phase II trial in completely resected stage IB and II NSCLC patients expressing MAGE-A3. 1089 patients were screened for MAGE-A3 expression, and 182 patients were included in the study and randomized either to be treated with the vaccine or placebo as an adjuvant treatment. The vaccine was administered 5 times at 3-week intervals, followed by maintenance therapy every 3 months for a total of 8 applications. In general, treatment was tolerated adequately with $9.6 \%$ of patients developing a grade 3 or 4 event, of which only 3 were considered related to the vaccine by the research team. After a follow-up of 28 months, $30.6 \%$ of patients in the vaccine arm and $43.3 \%$ in the placebo arm were diagnosed with a recurrence of the disease. DFS HR and OS HR were of 0.73 (95\% CI, $0.45-1.16 ; p=0.093)$ and 0.66 (95\% CI, $0.36-1.20 ; p=0.088)$ respectively. None of the study endpoint results between the arms were statistically significant [34]. A phase III study named MAGRIT (MAGE-A3 as Adjuvant non-small cell lunG cancer ImmunoTherapy) was initiated intended to recruit more than two thousand stage IB, II, or IIIA MAGE-A3 positive NSCLC patients. These will be randomized to either vaccine or placebo with DFS as the primary endpoint $[35,36]$.

\section{PF-3512676}

Other immunotherapeutic approaches have been investigated in the management of NSCLC.

PF-3512676 (ProMune; Coley Pharmaceutical Group), a toll-like receptor (TLR)-9 agonist was evaluated as an antigen-specific vaccine in a phase 2 study [37]. It has been proven that activation of TLR-9 may diminish the immune tolerance and ameliorate apoptosis and the recognition of tumor antigens [38]. In this study, 112 stage IIIB-IV NSCLC patients received chemotherapy and were then randomized to receive the vaccine or placebo. A trend of improvement in survival was seen in the vaccine arm with a higher myelosuppression seen in the same arm. Given the results of this trial, two phase III trials were started but later were discontinued since no improvement was seen in patients receiving the vaccine $[39,40]$.

\section{Belagenpumatucel-L}

Belagenpumatucel-L (Lucanix; NovaRx Corporation) tumor cell vaccine promotes the secretion of an antisense nucleotide to the transforming growth factor- $\beta 2$ (TGF$\beta 2)$. TGF- $\beta 2$ stimulates immunosuppression of natural killer cells and dendritic cell activity, and has also been identified as a marker of poor prognosis in NSCLC patients [41]. Preclinical studies showed that the inhibition of TGF- $\beta 2$ increased the immune response of tumor vaccines. A phase II study including 72 patients with stages II to IV NSCLC were randomized to one of three different dose levels $(1.25$, 2.5 or $5 \times 107$ cells per injection). There was a $16 \%$ response rate related to a higher amount of cells injected with a tolerable toxicity [42]. This vaccine has already entered a phase III trial intending to recruit around 500 stage IIIA (T3N2), IIIB, and IV NSCLC patients with overall survival as the primary endpoint [43].

\section{TG4010}

TG4010 also targets the MUC1 tumor associated antigen. In contrast to emepepimut-S, TG4010 also expresses IL-2, which has been demonstrated to reverse the suppression of T-cell response initiated by the MUC1 mucin [44]. A phase II open label trial included 148 MUC1 positive stage IIIB or IV untreated NSCLC patients. Patients were randomized to receive platinum-based chemotherapy with or without TG4010. The drug was given weekly for six weeks and then a maintenance therapy every three weeks until progression. PFS at six months was $44 \%$ versus $35 \%$ with a non-significant difference favoring the group receiving the vaccine. A statistical significant difference was seen in response rate $(43 \%$ versus $27 \%, p=0.03$ ) in those patients receiving the experimental drug [45]. A phase IIB/III study is waiting for 
participant recruitment, aiming for 1000 stage IV MUC1 positive NSCLC patients with PFS as the primary endpoint in the phase II study and OS in the phase III study [46].

\section{Anti-PD-1 Antibody}

Programmed death-1 (PD-1) protein is a T-cell receptor that interacts with 2 ligands, PD-L1 and PD-L2 expressed by tumor cells, stromal cells, or both where an immunosuppressive action is produced [47]. The inhibition of the interaction of the PD-1 receptor and the ligand therefore enhances T-cell-mediated immune responses [48]. BMS-936558, a human PD-1 specific antibody was developed and a phase I study with the drug included administered to a group of treatment refractory cancer patients, where 122 patients were NSCLC patients [49]. Patients were enrolled in either a 1.0, 3.0, or $10.0 \mathrm{mg}$ per kilogram of weight followed by a dose escalation protocol. Patients received the drug intravenously every 2 weeks in 8-week treatment cycle. Response rates with all doses were seen in $18 \%$ of patients with lung cancer and the majority of the responses were for a year or more. This study demonstrated a relationship between the antiPD-1 antibody and an objective response towards cancer.

\section{Conclusions}

Therapeutic vaccines for the treatment of NSCLC have been included in various clinical trials, hoping to obtain positive results in patients. Different mechanisms of action have been explored, including antigen-specific vaccines and tumor cell vaccines. Due to the high mortality rate in lung cancer, novel therapeutic options with a limi ted toxicity are needed to be developed. Unfortunately only a few of these compounds have been included in phase III trials, with L-BLP25 and MAGE-A3 being the most studied. These drugs have been used with a favorable toxicity profile which make them an interesting additional element to be included in the future treatment of NSCLC, including patient with a poor performance status. The results from these phase III trials are eagerly awaited hoping to verify the findings of previous trials.

These vaccines have included patients with NSCLC in early stages of the disease, and these are the patients that will probably benefit the most from this type of therapy. Locally advanced and metastatic patients have not showed a clear benefit from L-BLP25 or MAGE-A3 vaccines, and the reasons for this lack of response have not been elucidated. It has been observed that the immune response developed in patients treated with immunotherapy varies, even in those patients who showed a response. Therefore, other pathways may be involved in the response of these vaccines. As a result, biomarkers should be explored in order to identify which patients might develop a better immune response, and therefore show the most benefit from this type of therapy. Time will unveil the true importance and advantages of these vaccines or future vaccines as a treatment option in NSCLC patients.

\section{REFERENCES}

[1] L. Corrales-Rodriguez, N. Blais and D. Soulieres, "Emepepimut-S for Non-Small Cell Lung Cancer,” Expert Opinion on Biological Therapy, Vol. 11, No. 8, 2011, pp. 1091-1097. doi:10.1517/14712598.2011.592490

[2] F. Balkwill and A. Mantovani, "Inflammation and Cancer: Back to Virchow?” Lancet, Vol. 357, No. 9255, 2001, pp. 539-545. doi:10.1016/S0140-6736(00)04046-0

[3] L. M. Coussens and Z. Werb, "Inflammation and Cancer,” Nature, Vol. 420, No. 6917, 2002, pp. 860-867. doi:10.1038/nature01322

[4] P. A. Bradbury and F. A. Shepherd, "Immunotherapy for Lung Cancer,” Journal of Thoracic Oncology, Vol. 3, No. 6, 2008, pp. S164-S170. doi:10.1097/JTO.0b013e318174e9a7

[5] J. Banchereau and R. M. Steinman, "Dendritic Cells and the Control of Immunity,” Nature, Vol. 392, No. 6673, 1998, pp. 245-252.

[6] P. Bretscher and M. Cohn, "A theory of Self-Nonself Discrimination,” Science, Vol. 169, No. 3950, 1970, pp. 1042-1049. doi:10.1126/science.169.3950.1042

[7] A. Ribas, L. H. Butterfield, J. A. Glaspy and J. S. Economou, "Current Developments in Cancer Vaccines and Cellular Immunotherapy,” Journal of Clinical Oncology, Vol. 21, No. 12, 2003, pp. 2415-2432.

[8] J. Ferlay, H. R. Shin, F. Bray, D. Forman, C. Mathers and D. M. Parkin, "Estimates of Worldwide Burden of Cancer in 2008: GLOBOCAN 2008,” International Journal of Cancer, Vol. 127, No. 12, 2010, pp. 2893-2917. doi:10.1002/ijc.25516

[9] D. M. Parkin, F. Bray, J. Ferlay and P. Pisani, "Global Cancer Statistics, 2002,” Oncology \& Radiotherapy, Vol. 55, No. 2, 2005, pp. 74-108. doi:10.3322/canjclin.55.2.74

[10] F. C. Detterbeck, D. J. Boffa and L. T. Tanoue, "The New Lung Cancer Staging System,” Chest, Vol. 136, No. 1, 2009, pp. 260-271. doi:10.1378/chest.08-0978

[11] F. Bray, J. E. Tyczynski and D. M. Parkin, “Going Up or Coming Down? The Changing Phases of the Lung Cancer Epidemic from 1967 to 1999 in the 15 European Union Countries," European Journal of Cancer, Vol. 40, No. 1, 2004, pp. 96-125. doi:10.1016/j.ejca.2003.08.005

[12] P. J. Souquet, F. Chauvin, J. P. Boissel, R. Cellerino, Y. Cormier, P. A. Ganz, S. Kaasa, J. L. Pater, E. Quoix, E. Rapp, et al., "Polychemotherapy in Advanced Non Small Cell Lung Cancer: A Meta-Analysis,” Lancet, Vol. 342, No. 8862, 1993, pp. 19-21. doi:10.1016/0140-6736(93)91882-M

[13] A. Jemal, M. J. Thun, L. A. Ries, H. L. Howe, H. K. Weir, M. M. Center, E. Ward, X. C. Wu, C. Eheman, R. Anderson, U. A. Ajani, B. Kohler and B. K. Edwards, "Annual Report to the Nation on the Status of Cancer, 
1975-2005, Featuring Trends in Lung Cancer, Tobacco Use, and Tobacco Control," Journal of the National Cancer Institute, Vol. 100, No. 23, 2008, pp. 1672-1694. doi:10.1093/jnci/djn389

[14] S. Navaratnam, E. V. Kliewer, J. Butler, A. A. Demers, G. Musto and K. Badiani, "Population-Based Patterns and Cost of Management of Metastatic Non-Small Cell Lung Cancer after Completion of Chemotherapy Until Death,” Lung Cancer, Vol. 70, No. 1, 2010, pp. 110-115. doi:10.1016/j.lungcan.2010.01.012

[15] Food and Drug Administration, "Approval Letter-Provenge,” Food and Drug Administration, Silver Spring, 2010.

[16] P. W. Kantoff, C. S. Higano, N. D. Shore, E. R. Berger, E. J. Small, D. F. Penson, C. H. Redfern, A. C. Ferrari, R. Dreicer, R. B. Sims, Y. Xu, M. W. Frohlich and P. F. Schellhammer, "Sipuleucel-T Immunotherapy for Castration-Resistant Prostate Cancer," The New England Journal of Medicine, Vol. 363, No. 5, 2010, pp. 411-422. doi:10.1056/NEJMoa1001294

[17] C. Gridelli, A. Rossi, P. Maione, M. L. Ferrara, V. Castaldo and P. C. Sacco, "Vaccines for the Treatment of NonSmall Cell Lung Cancer: A Renewed Anticancer Strategy," Oncologist, Vol. 14, No. 9, 2009, pp. 909-920. doi:10.1634/theoncologist.2009-0017

[18] S. A. North, K. Graham, D. Bodnar and P. Venner, “A Pilot Study of the Liposomal MUC1 Vaccine BLP25 in Prostate Specific Antigen Failures after Radical Prostatectomy," Journal of Urology, Vol. 176, No. 1, 2006, pp. 9195. doi:10.1016/S0022-5347(06)00494-0

[19] R. Sangha and S. North, "L-BLP25: A MUC1-Targeted Peptide Vaccine Therapy in Prostate Cancer," Expert Opinion on Biological Therapy, Vol. 7, No. 11, 2007, pp. 1723-1730. doi:10.1517/14712598.7.11.1723

[20] B. Agrawal, M. J. Krantz, M. A. Reddish and B. M. Longenecker, "Rapid Induction of Primary Human CD4+ and CD8+ T Cell Responses against Cancer-Associated MUC1 Peptide Epitopes,” International Immunology, Vol. 10, No. 12, 1998, pp. 1907-1916. doi:10.1093/intimm/10.12.1907

[21] F. Ohyanagi, T. Horai, I. Sekine, N. Yamamoto, K. Nakagawa and M. Nishio, "L-BLP25 for Unresectable Stage III Non-Small Cell Lung Cancer after Primary Chemoradiotherapy in Japanese Patients: Preliminary Safety Results from a Phase I/II Study,” Journal of Clinical Oncology, Vol. 28, No. 15, 2010 , Article ID: e13153.

[22] C. Butts, N. Murray, A. Maksymiuk, G. Goss, E. Marshall, D. Soulieres, Y. Cormier, P. Ellis, A. Price, R. Sawhney, M. Davis, J. Mansi, C. Smith, D. Vergidis, P. Ellis, M. MacNeil and M. Palmer, "Randomized Phase IIB Trial of BLP25 Liposome Vaccine in Stage IIIB and IV Non-Small-Cell Lung Cancer,” Journal of Clinical Oncology, Vol. 23, No. 27, 2005, pp. 6674-6681.

[23] K. K. Bass and M. J. Mastrangelo, "Immunopotentiation with Low-Dose Cyclophosphamide in the Active Specific Immunotherapy of Cancer," Cancer Immunology, Immunotherapy, Vol. 47, No. 1, 1998, pp. 1-12. doi:10.1007/s002620050498

[24] G. D. MacLean, D. W. Miles, R. D. Rubens, M. A. Reddish and B. M. Longenecker, "Enhancing the Effect of
THERATOPE Stn-KLH Cancer Vaccine in Patients with Metastatic Breast Cancer by Pretreatment with Low-Dose Intravenous Cyclophosphamide,” Journal of Immunotherapy with Emphasis on Tumor Immunology, Vol. 19, No. 4, 1996, pp. 309-316.

[25] C. Butts, R. N. Murray, C. J. Smith, P. M. Ellis, K. Jasas, A. Maksymiuk, G. Goss, G. Ely, F. Beier and D. Soulieres, "A Multicenter Open-Label Study to Assess the Safety of a New Formulation of BLP25 Liposome Vaccine in Patients with Unresectable Stage III Non-SmallCell Lung Cancer," Clinical Lung Cancer, Vol. 11, No. 6, 2010, pp. 391-395. doi:10.3816/CLC.2010.n.101

[26] ClinicalTrials.gov, “Cancer Vaccine Study for Stage III, Unresectable, Non-Small Cell Lung Cancer (NSCLC) in the Asian Population (INSPIRE),” The U.S. National Institutes of Health, Bethesda, 2011.

[27] C. Butts, A. Maksymiuk, G. Goss, D. Soulieres, E. Marshall, Y. Cormier, P. Ellis, P. A. rice, R. Sawhney and N. Murray, "A Multi-Centre Phase IIB Randomized Controlled Study of BLP25 Liposome Vaccine (L-BLP25 or Stimuvax) for Active Specific Immunotherapy of NonSmall Cell Lung Cancer (NSCLC) Updated Survival Analysis,” Journal of Thoracic Oncology, Vol. 2, No. 8, 2007, pp. S332-S333.

[28] C. Butts, H. Anderson, A. Maksymiuk, D. Vergidis, D. Soulières, Y. Cormier, M. Davis, E. Marshall, M. Falk and G. Goss, "Long-term Safety of BLP25 Liposome Vaccine (L-BLP25) in Patients (Pts) with Stage IIIB/IV Non-Small Cell Lung Cancer(NSCLC)," Journal of Clinical Oncology, Vol. 27, No. S15, 2009.

[29] D. Soulieres, C. Smith, P. Ellis, N. Murray, K. Jasas, A. Maksymiuk, G. Goss, M. Falk and C. Butts, "A MultiCentre, Open-Label Study to Assess the Safety of Stimuvax (BLP25 Liposome Vaccine or L-BLP25) in NonSmall Cell Lung Cancer (NSCLC) Patients (Pts) with unresectable Stage III Disease,” Journal of Clinical Oncolo$g y$, Vol. 25, No. S18, 2007, p. 3075.

[30] http://www.emdserono.com/cmg.emdserono_us/en/image s/StimuvaxPR\%20-\%20FDA\%20lift\%20NSCLC\%20EM D\%20SERONO\%20VERSION\%20FINAL_tcm115_546 63.pdf

[31] B. Gaugler, B. Van den Eynde, P. van der Bruggen, P. Romero, J. J. Gaforio, E. De Plaen, B. Lethe, F. Brasseur and T. Boon, "Human Gene MAGE-3 Codes for an Antigen Recognized on a Melanoma by Autologous Cytolytic T Lymphocytes,” The Journal of Experimental Medicine, Vol. 179, No. 3, 1994, pp. 921-930. doi:10.1084/jem.179.3.921

[32] W. Sienel, C. Varwerk, A. Linder, D. Kaiser, M. Teschner, M. Delire, G. Stamatis and B. Passlick, "Melanoma Associated Antigen (MAGE)-A3 Expression in Stages I and II Non-Small Cell Lung Cancer: Results of a Multi-Center Study,” European Journal of Cardiothoracic Surgery, Vol. 25, No. 1, 2004, pp. 131-134. doi:10.1016/j.ejcts.2003.09.015

[33] W. H. Kruit, H. H. van Ojik, V. G. Brichard, B. Escudier, T. Dorval, B. Dreno, P. Patel, N. van Baren, M. F. Avril, S. Piperno, A. Khammari, M. Stas, G. Ritter, B. Lethe, D. Godelaine, F. Brasseur, Y. Zhang, P. van der Bruggen, T. Boon, A. M. Eggermont and M. Marchand, "Phase 1/2 
Study of Subcutaneous and Intradermal Immunization with a Recombinant MAGE-3 Protein in Patients with Detectable Metastatic Melanoma," International Journal of Cancer, Vol. 117, No. 4, 2005, pp. 596-604. doi:10.1002/ijc.21264

[34] J. Vansteenkiste, M. Zielinski, A. Linder, J. Dahabre, E. Esteban, W. Malinowski, J. Jassem, B. Passlick, F. Lehmann and V. Brichard, "Final Results of a Multi-Center, Double-Blind, Randomized, Placebo-Controlled Phase II Study to Assess the Efficacy of MAGE-A3 Immunotherapeutic as Adjuvant Therapy in Stage IB/II Non-Small Cell Lung Cancer (NSCLC),” Journal of Clinical Oncology, Vol. 25, No. S18, 2007, p. 7554.

[35] J. Vansteenkiste, M. Zielinski, I. Dahabreh, A. Linder, F. Lehmann, O. Gruselle, P. Therasse, J. Louahed and V. Brichard, "Association of Gene Expression Signature and Clinical Efficacy of MAGE-A3 Antigen-Specific Cancer Immunotherapeutic (ASCI) as Adjuvant Therapy in Resected Stage IB/II Non-Small Cell Lung Cancer (NSCLC)," Journal of Clinical Oncology, Vol. 26, No. S15, 2008.

[36] http://clinicaltrials.gov/ct2/show/NCT00480025

[37] C. Manegold, D. Gravenor, D. Woytowitz, et al., "Randomized Phase II Trial of a Toll-Like Receptor 9 Agonist Oligodeoxynucleotide, PF-3512676, in Combination with First-Line Taxane Plus Platinum Chemotherapy for Advanced-Stage Non-Small-Cell Lung Cancer," Journal of Clinical Oncology, Vol. 26, No. 24, 2008, pp. 3979-3986.

[38] H. Hemmi, O. Takeuchi, T. Kawai, T. Kaisho, S. Sato, et al., "Toll-Like Receptor Recognizes Bacterial DNA," Nature, Vol. 408, No. 6813, 2000, pp. 740-745. doi:10.1038/35047123

[39] C. Manegold, N. van Zandwijk, et al., "A Phase III Randomized Study of Gemcitabine and Cisplatin with or without PF-3512676 (TLR9 Agonist) as First-Line Treatment of Advanced Non-Small-Cell Lung Cancer," Annals of Oncology, Vol. 23, No. 1, 2012, pp. 72-77.

[40] V. Hirsh, L. Paz-Ares, et al., "Randomized Phase III Trial of Paclitaxel/Carboplatin with or without PF-3512676 (Toll-Like Receptor 9 Agonist) as First-Line Treatment for Advanced Non-Small-Cell Lung Cancer," Journal of Clinical Oncology, Vol. 29, No. 19, 2011, pp. 2667-2674.

[41] F. Kong, R. L. Jirtle, D. H. Huang, R. W. Clough and M. S. Anscher, "Plasma Transforming Growth Factor-Beta1
Level before Radiotherapy Correlates with Long Term Outcome of Patients with Lung Carcinoma," Cancer, Vol. 86, No. 9, 1999, pp. 1712-1719. doi:10.1002/(SICI)1097-0142(19991101)86:9<1712::AID -CNCR12>3.0.CO;2-V

[42] J. Nemunaitis, R. O. Dillman, et al., "Phase II Study of Belagenpumatucel-L, a Transforming Growth Factor Beta-2 Antisense Gene-Modified Allogeneic Tumor Cell Vaccine in Non-Small-Cell Lung Cancer," Journal of Clinical Oncology, Vol. 24, No. 29, 2006, pp. 4721-4730.

[43] "Phase III Lucanix ${ }^{\mathrm{TM}}$ Vaccine Therapy in Advanced Nonsmall Cell Lung Cancer (NSCLC) Following Front-line Chemotherapy (STOP).” http://clinicaltrials.gov/ct2/show/NCT00676507

[44] B. Agrawal, M. J. Krantz, M. A. Reddish and B. M. Longenecker, "Cancer-Associated MUC1 Mucin Inhibits Human T-Cell Proliferation, Which Is Reversible by IL-2," Nature Medicine, Vol. 4, No. 1, 1998, pp. 43-49. doi:10.1038/nm0198-043

[45] E. Quoix, R. Ramlau, V. Westeel, Z. Papai, A. Madroszyk, et al., "Therapeutic Vaccination with TG4010 and First-Line Chemotherapy in Advanced Non-Small-Cell Lung Cancer: A Controlled Phase 2B Trial,” The Lancet Oncology, Vol. 12, No. 12, 2011, pp. 1125-1133. doi:10.1016/S1470-2045(11)70259-5

[46] ClinicalTrials.gov, "Phase IIB/III of TG4010 Immunotherapy in Patients with Stage IV Non-Small Cell Lung Cancer (TIME),” 2012. http://clinicaltrials.gov/ct2/show/NCT01383148

[47] G. J. Freeman, A. J. Long, Y. Iwai, K. Bourque, T. Chernova, et al., "Engagement of the PD-1 Immunoinhibitory Receptor by a Novel B7 Family Member Leads to Negative Regulation of Lymphocyte Activation,” The Journal of Experimental Medicine, Vol. 192, No. 7, 2000, pp. 1027-1034.

[48] S. L. Topalian, C. G. Drake and D. M. Pardoll, "Targeting the PD-1/B7-H1 (PD-L1) Pathway to Activate AntiTumor Immunity,” Current Opinion in Immunology, Vol. 24, No. 2, 2012, pp. 207-212.

[49] S. L. Topalian, F. S. Hodi, J. R. Brahmer, S. N. Gettinger, et al., "Safety, Activity, and Immune Correlates of AntiPD-1 Antibody in Cancer," The New England Journal of Medicine, Vol. 366, No. 26, 2012, pp. 2443-2454. 